\title{
Shearingbox-implementation for the central-upwind, constraint-transport MHD-code NIRVANA
}

\author{
O. Gressel, U. Ziegler \\ Astrophysikalisches Institut Potsdam, D-14482 Potsdam, Germany
}

\begin{abstract}
We describe the implementation of the shearingbox approach into the Godunovtype central-upwind/constraint-transport magnetohydrodynamics code NIRVANA. This will allow for applications which require sheared-periodic boundary conditions as typically used in local Cartesian simulations of differentially rotating systems. We present the algorithm in detail and discuss necessary modifications in the numerical fluxes in order to preserve conserved quantities and to fulfill other analytical constraints as good as seem feasible within the numerical scheme. We check the source terms which come with the shearingbox formulation by investigating the conservation of the epicyclic mode energy. We also perform more realistic simulations of the magneto-rotational instability with initial zero-net-flux vertical magnetic field and compare the obtained stresses and energetics with previous non-conservative results exploring the same parameter regime.
\end{abstract}

Key words: Magnetohydrodynamics, Numerics: shearingbox

\section{Introduction}

Simulations aiming to study the local behavior of (magneto-)hydrodynamic flows usually invoke periodic boundary conditions. This accounts for the unboundedness of the flow and prevents the system from obtaining information about its surface. However, in the case of a shearing background flow periodicity is not strictly applicable. Typical scenarios for this include differentially rotating gaseous accretion disks, which are encountered in many astrophysical contexts. Such systems (if sufficiently ionized) are found to be unstable to the so-called magneto-rotational instability (MRI) and have been successfully modeled during the past decade (see [2,3] and references therein). Considerable progress in the field has been made using the local shearingbox approach, a detailed description of which can be found e.g. in [1]. 
The two key issues to be addressed in the design of shearingbox boundary conditions are (1) the time-dependent shifted-periodic mapping of the dependent variables of mass density, momentum, energy and, in case of magnetohydrodynamic flows, the magnetic field and (2) the offset introduced in the velocity component parallel to the shearing background. The latter represents the global shear across the domain which is forced constant in time. Both of these features and the numerical treatment of the resulting source terms arising in the local approximation have profound implications on the accuracy properties of the shearingbox advection/induction scheme.

In this paper we will deal with these implications in detail. First, section 2 gives a compact recollection of basic facts about the MHD scheme in NIRVANA necessary for a understanding of the following shearingbox implementation

which is then described in Section 3. Section 4 presents test cases to validate our approach.

\section{Review of the MHD scheme}

Although the NIRVANA code can account for dissipative effects (viscosity, diffusion, thermal heat conduction), multi-scale phenomena (using adaptive mesh refinement, see Ziegler [12]) as well as self-gravity (multigrid-type Poisson solver, see Ziegler [13]), we want to focus here on ideal MHD in three space dimensions described by the following set of coupled equations

$$
\begin{aligned}
\frac{\partial \mathbf{u}}{\partial t}+\frac{\partial \mathbf{f}^{x}}{\partial x}+\frac{\partial \mathbf{f}^{y}}{\partial y}+\frac{\partial \mathbf{f}^{z}}{\partial z} & =\mathbf{S}(\mathbf{u}) \\
\frac{\partial \mathbf{B}}{\partial t}+\nabla \times \mathbf{E} & =0
\end{aligned}
$$

where $\mathbf{u}=\left(\varrho, m_{x}, m_{y}, m_{z}, e\right)^{\top}$. The source term $\mathbf{S}$ arises from non-inertial forces in the local frame (rotating with $\Omega$ relative to the inertial frame) and will be discussed in detail in section 3.3. The flux functions $\mathbf{f}^{x}, \mathbf{f}^{y} . \mathbf{f}^{z}$ are defined as:

$$
\mathbf{f}^{x}=\left(\begin{array}{c}
m_{x} \\
m_{x} v_{x}+p+\frac{1}{2} \mathbf{B}^{2}-B_{x} B_{x} \\
m_{y} v_{x}-B_{y} B_{x} \\
m_{z} v_{x}-B_{z} B_{x} \\
\left(e+p+\frac{1}{2} \mathbf{B}^{2}\right) v_{x}-(\mathbf{v} \cdot \mathbf{B}) B_{x}
\end{array}\right)
$$




$$
\begin{gathered}
\mathbf{f}^{y}=\left(\begin{array}{c}
m_{y} \\
m_{x} v_{y}-B_{x} B_{x} \\
m_{y} v_{y}+p+\frac{1}{2} \mathbf{B}^{2}-B_{y} B_{y} \\
m_{z} v_{y}-B_{z} B_{y} \\
\left(e+p+\frac{1}{2} \mathbf{B}^{2}\right) v_{y}-(\mathbf{v} \cdot \mathbf{B}) B_{y}
\end{array}\right), \\
\mathbf{f}_{z}=\left(\begin{array}{c}
m_{x} v_{z}-B_{x} B_{z} \\
m_{y} v_{z}-B_{y} B_{z} \\
m_{z} v_{z}+p+\frac{1}{2} \mathbf{B}^{2}-B_{z} B_{z} \\
\left(e+p+\frac{1}{2} \mathbf{B}^{2}\right) v_{z}-(\mathbf{v} \cdot \mathbf{B}) B_{z}
\end{array}\right) .
\end{gathered}
$$

We adopt a notation where the magnetic permeability is set to unity. The equations are to be supplemented by the zero-divergence constraint $\nabla \cdot \mathbf{B}=0$ for the magnetic field $\mathbf{B}$ and an equation of state $e=\epsilon+\frac{1}{2} \varrho \mathbf{v}^{2}+\frac{1}{2} \mathbf{B}^{2}$ with $\epsilon=p /(\gamma-1)$. Other symbols have their usual meaning with $\varrho$ denoting the mass density, $e$ the total energy density, $\epsilon$ the thermal energy density, $p$ the pressure, $\mathbf{v}$ the velocity, $\mathbf{m}=\varrho \mathbf{v}$ the momentum, $\mathbf{E}=-\mathbf{v} \times \mathbf{B}$ the electric field and $\gamma$ the ratio of specific heats.

The numerical method used to solve the above system is a hybrid scheme combining the second-order version of the Godunov-type central-upwind scheme of Kurganov et al. [8] with the constraint transport (CT) technique for divergence free magnetic field evolution (see e.g. Evans \& Hawley [5], Tóth [10]). The original description of this hybrid approach can be found in Ziegler [11]. Here, we compactly summarize the relevant knowledge necessary for constructing the shearingbox-implementation.

Let the computational domain be subdivided in uniform Cartesian cells $C_{i, j, k}=$ $\left[x_{i-\frac{1}{2}}, x_{i+\frac{1}{2}}\right] \times\left[y_{j-\frac{1}{2}}, y_{j+\frac{1}{2}}\right] \times\left[z_{k-\frac{1}{2}}, z_{k+\frac{1}{2}}\right]$ with center $\left(x_{i}, y_{j}, z_{k}\right)$ and spacings $\delta x$, $\delta y$ and $\delta z$. Then, the scheme for Eq. (1) in semi-discrete form reads

$$
\begin{aligned}
\frac{d}{d t} \overline{\mathbf{u}}_{i, j, k}(t)= & -\frac{\mathbf{F}_{i+\frac{1}{2}, j, k}^{x}(t)-\mathbf{F}_{i-\frac{1}{2}, j, k}^{x}(t)}{\delta x}-\frac{\mathbf{F}_{i, j+\frac{1}{2}, k}^{y}(t)-\mathbf{F}_{i, j-\frac{1}{2}, k}^{y}(t)}{\delta y} \\
& -\frac{\mathbf{F}_{i, j, k+\frac{1}{2}}^{z}(t)-\mathbf{F}_{i, j, k-\frac{1}{2}}^{z}(t)}{\delta z}+\mathbf{S}\left(\overline{\mathbf{u}}_{i, j, k}(t)\right)
\end{aligned}
$$

where the cell-centered $\overline{\mathbf{u}}_{i, j, k}$ is an approximation to the volume-averaged state $\mathbf{u}$ in cell $C_{i, j, k}$ and the numerical fluxes are given by: 


$$
\begin{aligned}
& \mathbf{F}_{i+\frac{1}{2}, j, k}^{x}=\frac{a^{+} \mathbf{f}^{x}\left(\mathbf{w}_{i, j, k}^{E}\right)-a^{-} \mathbf{f}^{x}\left(\mathbf{w}_{i+1, j, k}^{W}\right)}{a^{+}-a^{-}}+\frac{a^{+} a^{-}\left(\mathbf{u}_{i+1, j, k}^{W}-\mathbf{u}_{i, j, k}^{E}\right)}{a^{+}-a^{-}}, \\
& \mathbf{F}_{i, j+\frac{1}{2}, k}^{y}=\frac{b^{+} \mathbf{f}^{y}\left(\mathbf{w}_{i, j, k}^{N}\right)-b^{-} \mathbf{f}^{y}\left(\mathbf{w}_{i, j+1, k}^{S}\right)}{b^{+}-b^{-}}+\frac{b^{+} b^{-}\left(\mathbf{u}_{i, j+1, k}^{S}-\mathbf{u}_{i, j, k}^{N}\right)}{b^{+}-b^{-}} \\
& \mathbf{F}_{i, j, k+\frac{1}{2}}^{z}=\frac{c^{+} \mathbf{f}^{z}\left(\mathbf{w}_{i, j, k}^{T}\right)-c^{-} \mathbf{f}^{z}\left(\mathbf{w}_{i, j, k+1}^{B}\right)}{c^{+}-c^{-}}+\frac{c^{+} c^{-}\left(\mathbf{u}_{i, j, k+1}^{B}-\mathbf{u}_{i, j, k}^{T}\right)}{c^{+}-c^{-}}
\end{aligned}
$$

with $\mathbf{w}=(\mathbf{u}, \mathbf{B})^{\top}$. We apply the third-order strong-stability-preserving RungeKutta scheme in [7] to integrate Eq. (6) in time (see also Eq. (19)). At any stage in the Runge-Kutta scheme the evaluation of the flux functions at cell faces requires point values for the variables left/right to a cell interface which are obtained by piecewise linear reconstruction from $\overline{\mathbf{w}}_{i, j, k}$ applying the TVD slope-limiter of van Leer [9]. These point values are characterized in the following by superscripts $\mathrm{W}$ at cell interface $x=x_{i-\frac{1}{2}}, \mathrm{E}$ at $x=x_{i+\frac{1}{2}}$, $\mathrm{S}$ at $y=y_{j-\frac{1}{2}}, \mathrm{~N}$ at $y=y_{j+\frac{1}{2}}, \mathrm{~B}$ at $z=z_{k-\frac{1}{2}}$ and $\mathrm{T}$ at $z=z_{k+\frac{1}{2}}$, respectively. With such notation $\mathbf{w}_{i+1, j, k}^{W}$ stands for the reconstructed value within cell $C_{i+1, j, k}$ at $x=x_{i+\frac{1}{2}}$ and $\mathbf{w}_{i, j, k}^{E}$ for the value within cell $C_{i, j, k}$ at the same position. In contrast to the cell-centered $\overline{\mathbf{u}}_{i, j, k}$ we use staggering for the magnetic field i.e. the face-centered finite-volume approximations to the face-averaged components are $\bar{B}_{x \mid i-\frac{1}{2}, j, k}, \bar{B}_{y \mid i, j-\frac{1}{2}, k}$ and $\bar{B}_{z \mid i, j, k-\frac{1}{2}}$, respectively. It follows that in the reconstruction of $\mathbf{B}$ at $\mathrm{E}, \mathrm{W}$ position the $\mathrm{x}$-component is already properly located, i.e. $\left(B_{x}\right)_{i, j, k}^{E, W}=\bar{B}_{x \mid i \pm \frac{1}{2}, j, k}$, whereas other components are first reconstructed in $x$-direction and then spatially averaged in the transverse direction. Reconstruction of $\mathbf{B}$ at the other locations N,S,B,T is done in analogous fashion.

The quantities $a^{ \pm}$in the flux formulae define the maximum (plus sign) respective minimum (minus sign) wave-propagation-direction-sensitive speed at the interface $x_{i+\frac{1}{2}}$, i.e.

$$
\begin{aligned}
& a^{+}=\max \left\{\left(v_{x}+c_{\mathrm{f}}\right)_{i+1, j, k}^{W},\left(v_{x}+c_{\mathrm{f}}\right)_{i, j, k}^{E}, 0\right\}, \\
& a^{-}=\min \left\{\left(v_{x}-c_{\mathrm{f}}\right)_{i+1, j, k}^{W},\left(v_{x}-c_{\mathrm{f}}\right)_{i, j, k}^{E}, 0\right\}
\end{aligned}
$$

where $c_{\mathrm{f}}=\left(c_{S}^{2}+c_{A}^{2}\right)^{1 / 2}$ is an upper limit for the fast magnetosonic speed including the sound speed $c_{\mathrm{s}}=(\gamma p / \varrho)^{1 / 2}$ and Alfvén-speed $c_{A}=\left(\mathbf{B}^{2} / \varrho\right)^{1 / 2}$. The corresponding speeds in the $y(z)$-direction are denoted by $b^{ \pm}\left(c^{ \pm}\right)$.

The above method can be formally applied to the induction equation (2). 
Writing the curl of the electric field as divergence of an antisymmetric tensor

$$
-\nabla \times \mathbf{E}=\nabla \cdot\left(\begin{array}{ccc}
0 & E_{z} & -E_{y} \\
-E_{z} & 0 & E_{x} \\
E_{y} & -E_{x} & 0
\end{array}\right)
$$

and utilizing the abbreviations $\varepsilon^{x}=\left(0,-E_{z}, E_{y}\right)^{\top}, \varepsilon^{y}=\left(E_{z}, 0,-E_{x}\right)^{\top}$, and $\varepsilon^{z}=\left(-E_{y}, E_{x}, 0\right)^{\top}$, in analogy to Eqs. (7)-(9) one can derive electric field fluxes

$$
\begin{aligned}
& \mathbf{G}_{i+\frac{1}{2}, j, k}^{x}=\frac{a^{+} \varepsilon^{x}\left(\mathbf{w}_{i, j, k}^{E}\right)-a^{-} \varepsilon^{x}\left(\mathbf{w}_{i+1, j, k}^{W}\right)}{a^{+}-a^{-}}+\frac{a^{+} a^{-}\left(\mathbf{B}_{i+1, j, k}^{W}-\mathbf{B}_{i, j, k}^{E}\right)}{a^{+}-a^{-}} \\
& \mathbf{G}_{i, j+\frac{1}{2}, k}^{y}=\frac{b^{+} \boldsymbol{\varepsilon}^{y}\left(\mathbf{w}_{i, j, k}^{N}\right)-b^{-} \boldsymbol{\varepsilon}^{y}\left(\mathbf{w}_{i, j+1, k}^{S}\right)}{b^{+}-b^{-}}+\frac{b^{+} b^{-}\left(\mathbf{B}_{i, j+1, k}^{S}-\mathbf{B}_{i, j, k}^{N}\right)}{b^{+}-b^{-}} \\
& \mathbf{G}_{i, j, k+\frac{1}{2}}^{z}=\frac{c^{+} \boldsymbol{\varepsilon}^{z}\left(\mathbf{w}_{i, j, k}^{T}\right)-c^{-} \boldsymbol{\varepsilon}^{z}\left(\mathbf{w}_{i, j, k+1}^{B}\right)}{c^{+}-c^{-}}+\frac{c^{+} c^{-}\left(\mathbf{B}_{i, j, k+1}^{B}-\mathbf{B}_{i, j, k}^{T}\right)}{c^{+}-c^{-}}
\end{aligned}
$$

These are, like the F-fluxes, defined at cell faces. As before, at any stage in the Runge-Kutta scheme, the electric field function $\varepsilon^{x}\left(\varepsilon^{y}, \varepsilon^{z}\right)$ have to be evaluated at E,W (N,S,T,B) cell interfaces using reconstructed data, e.g., $\varepsilon_{y}^{x}\left(\mathbf{w}_{i, j, k}^{E}\right)=\left(-E_{z}\right)_{i, j, k}^{E}=-\left(v_{x}\right)_{i, j, k}^{E}\left(B_{y}\right)_{i, j, k}^{E}+\left(v_{y}\right)_{i, j, k}^{E}\left(B_{x}\right)_{i, j, k}^{E}$. Our CT scheme for the staggered magnetic field components finally reads in semi-discrete form

$$
\begin{aligned}
\frac{d}{d t} \bar{B}_{x \mid i-\frac{1}{2}, j, k} & =-\frac{\bar{E}_{z \mid i-\frac{1}{2}, j+\frac{1}{2}, k}-\bar{E}_{z \mid i-\frac{1}{2}, j-\frac{1}{2}, k}}{\delta y}+\frac{\bar{E}_{y \mid i-\frac{1}{2}, j, k+\frac{1}{2}}-\bar{E}_{y \mid i-\frac{1}{2}, j, k-\frac{1}{2}}}{\delta z}, \\
\frac{d}{d t} \bar{B}_{y \mid i, j-\frac{1}{2}, k} & =+\frac{\bar{E}_{z \mid i+\frac{1}{2}, j-\frac{1}{2}, k}-\bar{E}_{z \mid i-\frac{1}{2}, j-\frac{1}{2}, k}}{\delta x}-\frac{\bar{E}_{x \mid i, j-\frac{1}{2}, k+\frac{1}{2}}-\bar{E}_{x \mid i, j-\frac{1}{2}, k-\frac{1}{2}}}{\delta z} \\
\frac{d}{d t} \bar{B}_{z \mid i, j, k-\frac{1}{2}} & =-\frac{\bar{E}_{y \mid i+\frac{1}{2}, j, k-\frac{1}{2}}-\bar{E}_{y \mid i-\frac{1}{2}, j, k-\frac{1}{2}}}{\delta x}+\frac{\bar{E}_{x \mid i, j+\frac{1}{2}, k-\frac{1}{2}}-\bar{E}_{x \mid i, j-\frac{1}{2}, k-\frac{1}{2}}}{\delta y}
\end{aligned}
$$

where $\bar{E}_{x \mid i, j-\frac{1}{2}, k-\frac{1}{2}}, \bar{E}_{y \mid i-\frac{1}{2}, j, k-\frac{1}{2}}, \bar{E}_{z \mid i-\frac{1}{2}, j-\frac{1}{2}, k}$ are edge-centered approximations to the edge-averaged electric field components. These are computed by composition of the electric field fluxes obtained from the Godunov-central scheme, i.e.,

$$
\bar{E}_{x \mid i, j-\frac{1}{2}, k-\frac{1}{2}}=\frac{1}{4}\left(-\mathbf{G}_{z \mid i, j-\frac{1}{2}, k}^{y}-\mathbf{G}_{z \mid i, j-\frac{1}{2}, k-1}^{y}+\mathbf{G}_{y \mid i, j, k-\frac{1}{2}}^{z}+\mathbf{G}_{y \mid i, j-1, k-\frac{1}{2}}^{z}\right),
$$




$$
\begin{aligned}
& \bar{E}_{y \mid i-\frac{1}{2}, j, k-\frac{1}{2}}=\frac{1}{4}\left(+\mathbf{G}_{z \mid i-\frac{1}{2}, j, k}^{x}+\mathbf{G}_{z \mid i-\frac{1}{2}, j, k-1}^{x}-\mathbf{G}_{x \mid i, j, k-\frac{1}{2}}^{z}-\mathbf{G}_{x \mid i-1, j, k-\frac{1}{2}}^{z}\right), \\
& \bar{E}_{z \mid i-\frac{1}{2}, j-\frac{1}{2}, k}=\frac{1}{4}\left(-\mathbf{G}_{y \mid i-\frac{1}{2}, j, k}^{x}-\mathbf{G}_{y \mid i-\frac{1}{2}, j-1, k}^{x}+\mathbf{G}_{x \mid i, j-\frac{1}{2}, k}^{y}+\mathbf{G}_{x \mid i-1, j-\frac{1}{2}, k}^{y}\right) .
\end{aligned}
$$

It is now easy to show from $(13)-(15)$ that

$$
\begin{aligned}
\frac{d}{d t}(\nabla \cdot \overline{\mathbf{B}})_{i, j, k}=\frac{d}{d t} & \left(\frac{\bar{B}_{x \mid i+\frac{1}{2}, j, k}-\bar{B}_{x \mid i-\frac{1}{2}, j, k}}{\delta x}\right. \\
& \left.+\frac{\bar{B}_{y \mid i, j+\frac{1}{2}, k}-\bar{B}_{y \mid i, j-\frac{1}{2}, k}}{\delta y}+\frac{\bar{B}_{z \mid i, j, k+\frac{1}{2}}-\bar{B}_{z \mid i, j, k-\frac{1}{2}}}{\delta z}\right)=0 .
\end{aligned}
$$

Thus, if $(\nabla \cdot \overline{\mathbf{B}})_{i, j, k}=0$ initially, the system evolves divergence-free since this condition is fulfilled after each stage in the multi-stage Runge-Kutta scheme.

The full system of ODEs (6), (13)-(15) including the source term is solved with the strong-stability-preserving third-order Runge-Kutta integration scheme given by (dropping cell indices and writing the rhs of Eq. 6 as $\mathbf{L}_{F}+\mathbf{S}$ and (13)-(15) compactely as $\frac{d}{d t} \overline{\mathbf{B}}=\mathbf{L}_{E}$ )

$$
\begin{aligned}
\overline{\mathbf{u}}^{(1)} & =\overline{\mathbf{u}}^{n}+\delta t\left(\mathbf{L}_{F}^{n}+\mathbf{S}^{n}\right) \\
\overline{\mathbf{B}}^{(1)} & =\overline{\mathbf{B}}^{n}+\delta t \mathbf{L}_{E}^{n}, \\
\overline{\mathbf{u}}^{(2)} & =\frac{3}{4} \overline{\mathbf{u}}^{n}+\frac{1}{4} \overline{\mathbf{u}}^{(1)}+\frac{1}{4} \delta t\left(\mathbf{L}_{F}^{(1)}+\mathbf{S}^{(1)}\right) \\
\overline{\mathbf{B}}^{(2)} & =\frac{3}{4} \overline{\mathbf{B}}^{n}+\frac{1}{4} \overline{\mathbf{B}}^{(1)}+\frac{1}{4} \delta t \mathbf{L}_{E}^{(1)}, \\
\overline{\mathbf{u}}^{n+1} & =\frac{1}{3} \overline{\mathbf{u}}^{n}+\frac{2}{3} \overline{\mathbf{u}}^{(2)}+\frac{2}{3} \delta t\left(\mathbf{L}_{F}^{(2)}+\mathbf{S}^{(2)}\right) \\
\overline{\mathbf{B}}^{n+1} & =\frac{1}{3} \overline{\mathbf{B}}^{n}+\frac{2}{3} \overline{\mathbf{B}}^{(2)}+\frac{2}{3} \delta t \mathbf{L}_{E}^{(2)}
\end{aligned}
$$

with the time-step $\delta t=t^{n+1}-t^{n}$ restricted by the CFL condition.

\section{Shearingbox implementation}

Let us now consider a magnetohydrodynamic flow with a background profile of the form $\mathbf{v}=\left(0, v_{y}=s x, 0\right)$, with (linear) shear-parameter $s$. The computational domain is a Cartesian box with dimensions $L_{x}, L_{y}, L_{z}$ and periodic boundaries in $y$ - and $z$-direction. To account for the background shear we 


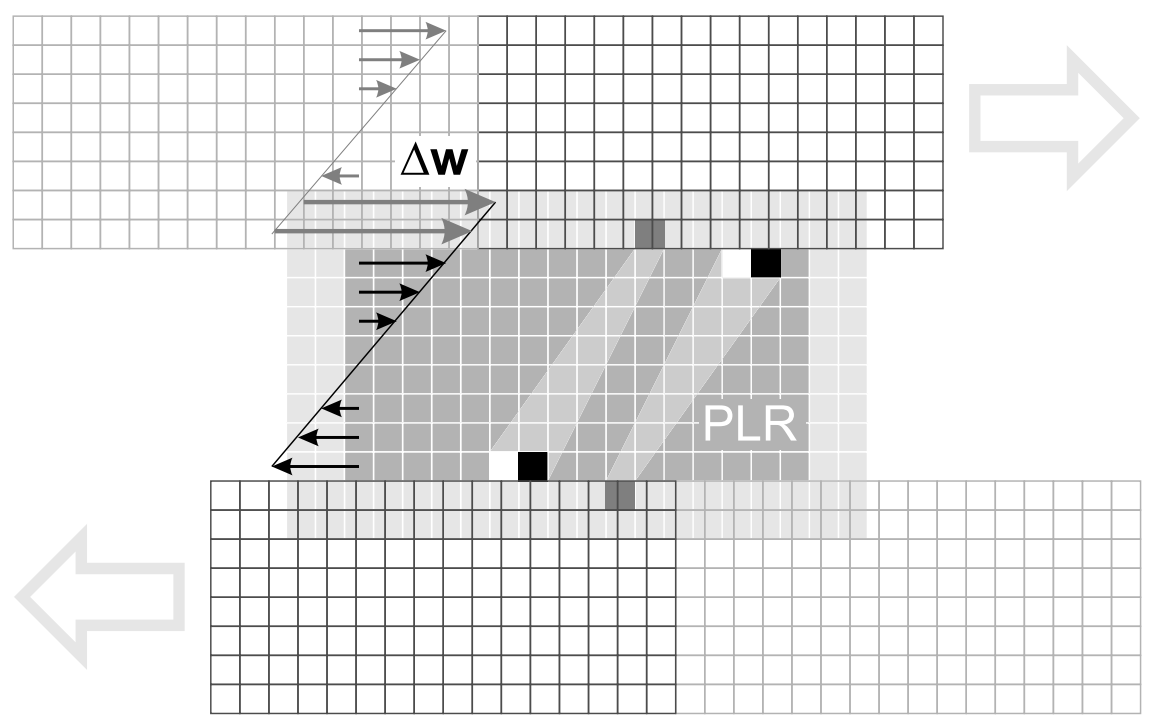

Fig. 1. Mapping of ghost zone values for a 2D shearingsheet. Reconstruction is indicated by highlighted cells. Arrows illustrate the need for a global velocity offset to match the velocity profile of the neighboring domain.

introduce shearingbox boundary conditions in $x$-direction. These can be expressed mathematically in the form ${ }^{1}$ (e.g. [1]):

$$
\begin{aligned}
& f(x, y, z) \mapsto f\left(x \pm L_{x}, y \mp w t, z\right), \quad f \in\left\{\varrho, m_{x}, m_{z}, \epsilon\right\} \\
& m_{y}(x, y, z) \mapsto m_{y}\left(x \pm L_{x}, y \mp w t, z\right) \mp \varrho w,
\end{aligned}
$$

where $w=-s L_{x}$ represents the global velocity-offset across the box, as can be seen in Figure 1. Because NIRVANA being a conservative code evolves the total energy (rather than the internal energy $\epsilon$ ) we supplement the corresponding relation for the total energy density

$$
e(x, y, z) \mapsto e\left(x \pm L_{x}, y \mp w t, z\right) \mp m_{y} w+\frac{1}{2} \varrho w^{2}
$$

which can be easily derived by separating the $m_{y}^{2} /(2 \varrho)$ part from the total energy and then using (21).

As the $y$-coordinate of above mappings varies continously in time, there is some kind of interpolation necessary to map ghostzone values on a finite grid (see e.g. [1] for details). For our implementation, the same piecewise linear reconstruction is used as for the numerical scheme.

Due to the shifted periodicity and the additional interpolation, ghost cells on opposing sides of the domain along with their adjoint regular zones are not re-

$\overline{1}$ Position arguments are being suppressed for extra variables in eqs. (21), (22). 
dundant in the same sense as for strictly periodic boundary conditions. Let us elucidate on that: In the case of regular periodicity every pair of cell and ghost cell (separated by the domain boundary) has an identical counterpart at the opposite boundary. Therefore the total flux across the interface is conserved to machine accuracy.

For shifted periodicity at a given time $t$ this does no longer hold. Thus the additional truncation error connected to the interpolation can lead to the buildup of considerable deviations in conserved quantities, as is shown in section 4.1. In the following we want to suggest a method to correct for this. Since strict conservation only applies as long as there is no source on the rhs of (1), in the local shearingbox approach with source term $(33) /(34)$ only the total zmomentum and mass are exactly conserved. We note that it is nevertheless desirable to preserve all variables as good as possible under advection.

\subsection{Conservation of hydrodynamic variables}

As the numerical fluxes are nonlinear functions of the conserved quantities any form of interpolation for the ghost cells will lead to some small inconsistency in the fluxes. This can be avoided by matching the computed $x$-fluxes at the sheared domain boundaries. It is straightforward to map fluxes not containing $m_{y}$. The quantities related to those fluxes, i.e. mass density, $x$ and $z$-momentum are then conserved to machine precision with respect to advection. For the $y$-momentum flux and total energy flux, applying the mappings $(21) /(22)$ to the third and fifth component of the flux function (3) yields:

$$
\begin{aligned}
f^{x}\left(m_{y}\right) & \mapsto f^{x}\left(m_{y}\right) \mp f^{x}(\varrho) w \\
f^{x}(e) & \mapsto f^{x}(e) \mp f^{x}\left(m_{y}\right) w+\frac{1}{2} f^{x}(\varrho) w^{2}
\end{aligned}
$$

in nice analogy to the original mappings. We want to point out that these relations consistently include the magnetic part of the fluxes due to the Lorentzforce, although this is not directly visible in the above notation. Also, the modifications to the fluxes can be solely expressed in terms of fluxes and the velocity offset $w$.

The recipe can then directly be carried over to the numerical fluxes (7): while the first term is just a linear combination of the flux-function (evaluated at the adequate positions) one has to apply $(21) /(22)$ once again for the second term, which then gives:

$$
F_{i+\frac{1}{2}, j, k}^{x}\left(m_{y}\right)=\widehat{F}_{\tilde{\imath}+\frac{1}{2}, \tilde{\jmath}, k}^{x}\left(m_{y}\right) \mp \widehat{F}_{\tilde{\imath}+\frac{1}{2}, \tilde{\jmath}, k}^{x}(\varrho) w
$$




$$
F_{i+\frac{1}{2}, j, k}^{x}(e)=\widehat{F}_{\tilde{\imath}+\frac{1}{2}, \tilde{\jmath}, k}^{x}(e) \mp \widehat{F}_{\tilde{\imath}+\frac{1}{2}, \tilde{\jmath}, k}^{x}\left(m_{y}\right) w+\frac{1}{2} \widehat{F}_{\tilde{\imath}+\frac{1}{2}, \tilde{\jmath}, k}^{x}(\varrho) w^{2},
$$

where the hat stands for the piecewise linear interpolation procedure used, and tilde marks the corresponding indices of the zones to map from.

Relation (24) expresses the fact that the total energy within the shearingbox is not conserved but can be altered by angular momentum transport through the radial boundaries. In integral formulation this corresponds to Eq. (8) (cast into our notation) of Hawley et al. [1]:

$$
\frac{\partial \Gamma}{\partial t}=w \int_{\partial X} \mathrm{dydz}\left(\varrho v_{x} \delta v_{y}-B_{x} B_{y}\right)
$$

where $\Gamma$ is the volume integral of total energy and $\delta v_{y}=v_{y}+s x$ expresses the perturbed velocity. ${ }^{2}$ When correcting the energy- and momentum-fluxes according to Eqs. (23), (24) one can satisfy this property more accurately which allows to trace the detailed evolution of energetics, e.g. in MRI-simulations.

\subsection{Conservation of magnetic flux}

Applying Gauß' theorem to the integral form of the induction equation, one can show that the azimuthal field (due to the shear) grows linearly with the net radial magnetic field-flux through the radial boundaries:

$$
\frac{\partial\langle\mathbf{B}\rangle}{\partial t}=-\frac{w}{V} \hat{\mathbf{y}} \int_{\partial X} \operatorname{dydz} B_{x} .
$$

For zero net radial field the mean magnetic flux through the shearingbox is conserved. Our implementation satisfies this condition to machine accuracy for the $x$ - and $z$-component of the magnetic field and to truncation error for the $y$-component (see section 4.1). This corresponds to the amount of precision reported by $[1,6]$.

The seminal paper of Hawley et. al [1] discusses this topic rather briefly, mentioning that mapping the electromotive forces at the sheared interfaces conserves the vertical field to roundoff error. Furthermore, the authors state that the spurious vertical field, that arises otherwise, is negligible as the associated MRI growth rates are unresolved. Considering the differences between MRI-simulations with and without zero net vertical field (see e.g. [6]) leaves

$\overline{2}$ Expanding $\delta v_{y}$ yields the additional $w^{2}$ term in (24). 
this somewhat questionable in the sense that a spurious field might lead to an overestimation of magnetic stresses in the latter case. One might further investigate this by a series of MRI-simulations with initial vertical field of the form $B_{z}=B_{0} \sin \left(2 \pi x / L_{x}\right)+B_{1}$, where $\left|B_{1}\right| \ll\left|B_{0}\right|$, i.e., with a controlled "spurious" field. Although from the physical point of view an exactly vanishing vertical flux is rather a question of academic nature, the issue of enhanced turbulent transport for net vertical field is still standing.

In our implementation we apply additional boundary conditions to the electric field fluxes $\mathbf{G}$ to assure conservation of mean magnetic fields. Due to the different staggering of magnetic field components, which are face-centered, the method has to be applied for the fluxes in all three directions. The velocity offset $w$ enters the fluxes via the electromotive force:

$$
\mathbf{E}(x, y, z) \mapsto \mathbf{E}\left(x \pm L_{x}, y \mp w t, z\right) \pm w \hat{\mathbf{y}} \times \mathbf{B}
$$

For the $x(z)$-direction only the $x(z)$-component of the magnetic field is needed to evaluate the additional second term. As the staggering of the normal field component coincides with that of the fluxes no reconstruction is needed in this case. Employing $B_{x \mid i, j, k}^{E}=B_{x \mid i+1, j, k}^{W}$ (respectively $B_{z \mid i, j, k}^{T}=B_{z \mid i, j, k+1}^{B}$ ) the characteristic velocities cancel out and we yield

$$
\begin{aligned}
\mathbf{G}_{i+\frac{1}{2}, j, k}^{x} & =\widehat{\mathbf{G}}_{\tilde{\imath}+\frac{1}{2}, \tilde{\jmath}, k}^{x} \mp w \hat{\mathbf{y}} B_{x \mid \tilde{\imath}+\frac{1}{2}, \tilde{\jmath}, k} \\
\mathbf{G}_{i, j, k+\frac{1}{2}}^{z} & =\widehat{\mathbf{G}}_{\tilde{\imath}, \tilde{\jmath}, k+\frac{1}{2}}^{z} \mp w \hat{\mathbf{y}} B_{z \mid \tilde{\imath}, \tilde{\jmath}, k+\frac{1}{2}}
\end{aligned}
$$

for the numerical electric fluxes in $x / z$-direction. For the remaining direction matters are a bit more complicated and we need the full reconstruction to be consistent with the underlying numerical scheme:

$$
\mathbf{G}_{i, j+\frac{1}{2}, k}^{y}=\widehat{\mathbf{G}}_{\tilde{\imath}, \tilde{\jmath}+\frac{1}{2}, k}^{y} \pm w \frac{b^{+}\left(B_{x} \hat{\mathbf{x}}+B_{z} \hat{\mathbf{z}}\right)_{\tilde{\imath}, \tilde{\jmath}, k}^{N}-b^{-}\left(B_{x} \hat{\mathbf{x}}+B_{z} \hat{\mathbf{z}}\right)_{\tilde{\imath}, \tilde{\jmath}, k}^{S}}{b^{+}-b^{-}}
$$

To conclude the discussion of the boundary conditions we want to remark that the reconstruction of the magnetic field at the sheared interfaces preserves the $\nabla \cdot \overline{\mathbf{B}}=0$ constraint to roundoff error (see section 4.1 ). The actual code is publicly available on the internet at www.aip.de/ gressel/index.php?id=code and can be embedded into the original NIRVANA package.

The current implementation supports distributed memory parallelism in the form of blockwise domain decomposition along the z-coordinate. In this case all the information needed to reconstruct the boundary values is available locally, i.e., without MPI communications. Additional block distribution along the $\mathrm{x}$ - 
and y-coordinates would in principle improve the surface to volume ratio but would also add communication overhead. This is discussed in detail in sections 4.3 and 4.4 of [4]. Their Figure 5 shows that block distribution along the $\mathrm{x}$-coordinate is inefficient and performance gains for additional distribution along the y-direction are moderate. They argue that the poor performance for $\mathrm{x}$-distribution might be a cache issue with the Fortran column major ordering, which leaves this result marginally meaningful for our implementation. In the case of vertically elongated boxes, like they are common for simulations in galactic environments, distribution along $\mathrm{z}$ even outperforms $\mathrm{y}$ - and $\mathrm{y}-\mathrm{z}-$ distributions.

\subsection{Source terms}

The second substantial ingredient of the shearingbox formalism are the source terms in the so called Hill system. This approximation is based on the local expansion of the equations of motion resulting in a tidal force $2 q \Omega^{2} x$ where $q=\mathrm{d} \ln \Omega / \mathrm{d} \ln R$ represents the (angular) shear parameter for a differential rotation of the form $\Omega(R) \propto R^{-q}$. Together with the Coriolis force the source terms for the momentum- and energy equation are:

$$
\begin{aligned}
S(\mathbf{m}) & =-2 \varrho \Omega \hat{\mathbf{z}} \times \mathbf{v}+2 \varrho q \Omega^{2} x \hat{\mathbf{x}}=-2 \varrho \Omega \hat{\mathbf{z}} \times(\mathbf{v}+q \Omega x \hat{\mathbf{y}}) \\
S(e) & =+2 \varrho \Omega^{2} q x \hat{\mathbf{x}} \cdot \mathbf{v}
\end{aligned}
$$

Equation (33) shows that the momentum source terms can be combined and act as an effective ${ }^{3}$ Coriolis force on the perturbed velocity $\delta v_{y}=v_{y}+q \Omega x$. This would in principle allow for an exact Coriolis-update in the form of an analytic rotation. Numerical tests, however, show that such an update is not suitable for the multi-stage Runge-Kutta integration scheme. Because operator-splitting is not favorable (for reasons described in section 4.2 below) we decide to implement the source terms unsplit, i.e., as explicit forces within the Runge-Kutta time integration scheme (see eq. (19)).

Gardiner \& Stone [6] stress the importance of conserving the energy contained in the epicyclic mode. This ideally conserved quantity can be derived from the energy budget in the limit of inviscid flow. The general expression reads:

$$
E_{\mathrm{e} p i}=\frac{1}{2} \varrho\left\langle u_{R}\right\rangle^{2}+\frac{(2 \Omega)^{2}}{\kappa^{2}} \frac{1}{2} \varrho\left\langle u_{\phi}\right\rangle^{2}=\frac{1}{2} \varrho\left(\left\langle u_{R}\right\rangle^{2}+\frac{2}{2-q}\left\langle u_{\phi}\right\rangle^{2}\right),
$$

$\overline{3}$ In contrast to the description in the Lagrangian frame of reference the angular velocity $\Omega=\Omega_{0}$ is not a function of radius here. 


\begin{tabular}{|l|c|c|c|c|c|c|}
\hline resolution & & $24^{2}$ & $32^{2}$ & $48^{2}$ & $64^{2}$ & $96^{2}$ \\
\hline \hline mass & a & $1.3371 \mathrm{e}-07$ & $1.2407 \mathrm{e}-07$ & $6.0259 \mathrm{e}-08$ & $1.2674 \mathrm{e}-08$ & $8.5488 \mathrm{e}-09$ \\
\hline & $\mathrm{b}$ & $4.8018 \mathrm{e}-16$ & $1.1181 \mathrm{e}-15$ & $8.0027 \mathrm{e}-16$ & $1.4405 \mathrm{e}-15$ & $1.6005 \mathrm{e}-15$ \\
\hline
\end{tabular}

Table 1

Convergence study of the relative error in total mass: (a) without special treatment, (b) with the method described above.

with $\kappa$ the epicyclic frequency. This formally looks like a kinetic energy but also includes the potential energy with respect to the epicyclic displacement. We have found that it is important to implement the source terms in an unsplit fashion to avoid oscillations in this energy that would otherwise arise from systematic splitting errors. This is discussed in more detail in section 4.2 below.

\section{Test cases}

We have validated our implementation with various simple advection tests, i.e., with source terms switched off, and have checked the mentioned conservation properties.

\subsection{Advection tests}

As an example Table 1 shows the maximum relative error in the total mass contained in our simulation box. Without special treatment of the fluxes we find an error that decreases with increasing resolution, i.e., has the form of a truncation error. With our modified treatment the total mass is conserved to roundoff error. Albeit not explicitely shown in Table 1, this is also true for the momentum and total energy.

As discussed in section 3.2, for initially zero mean radial field all components of the mean magnetic field are ideally conserved. In Table 2 we show the components of the mean magnetic field, normalized to corresponding rms values. One can see that without proper mapping of the field fluxes the analytic constraint is only fullfilled to roundoff error for $B_{x}$. With the method described above one can also conserve the $B_{z}$ component to machine accuracy, while the error in the $B_{y}$ component can be reduced by about an order of magnitude. As can also be seen from Table 2, the maximum error in the solenoidal constraint is of the order $10^{-13}$ while the average error is as low as $5 \times 10^{-15}$. For the state of fully developed turbulence in the MRI-simulations (see section 4.3) the max./avg. values rise to $10^{-10}$ and $10^{-13}$, respectively. 
Table 2

\begin{tabular}{|l|c|c|c|c|c|}
\hline resolution & & $24^{3}$ & $32^{3}$ & $48^{3}$ & $64^{3}$ \\
\hline \hline$\left\langle B_{x}\right\rangle / B_{x}^{\text {rms }}$ & a & $9.800 \mathrm{e}-16$ & $1.062 \mathrm{e}-15$ & $1.426 \mathrm{e}-15$ & $5.285 \mathrm{e}-15$ \\
\hline & b & $1.292 \mathrm{e}-15$ & $1.512 \mathrm{e}-15$ & $2.086 \mathrm{e}-15$ & $5.658 \mathrm{e}-15$ \\
\hline$\left\langle B_{y}\right\rangle / B_{y}^{\text {rms }}$ & a & $1.043 \mathrm{e}-05$ & $3.085 \mathrm{e}-06$ & $4.021 \mathrm{e}-06$ & $2.600 \mathrm{e}-06$ \\
\hline & b & $2.454 \mathrm{e}-06$ & $1.327 \mathrm{e}-06$ & $4.000 \mathrm{e}-07$ & $1.008 \mathrm{e}-07$ \\
\hline$\left\langle B_{z}\right\rangle / B_{z}^{\text {rms }}$ & a & $1.314 \mathrm{e}-05$ & $1.602 \mathrm{e}-06$ & $8.545 \mathrm{e}-06$ & $7.646 \mathrm{e}-06$ \\
\hline & b & $4.698 \mathrm{e}-17$ & $4.574 \mathrm{e}-17$ & $4.680 \mathrm{e}-17$ & $7.304 \mathrm{e}-17$ \\
\hline max. $\nabla \cdot \mathbf{B} /|\mathbf{B}|$ & a & $3.854 \mathrm{e}-13$ & $3.448 \mathrm{e}-13$ & $7.446 \mathrm{e}-13$ & $5.671 \mathrm{e}-13$ \\
\hline & b & $5.721 \mathrm{e}-13$ & $1.879 \mathrm{e}-13$ & $1.016 \mathrm{e}-12$ & $1.177 \mathrm{e}-12$ \\
\hline avg. $\nabla \cdot \mathbf{B} /|\mathbf{B}|$ & a & $5.493 \mathrm{e}-15$ & $5.469 \mathrm{e}-15$ & $4.575 \mathrm{e}-15$ & $3.964 \mathrm{e}-15$ \\
\hline & b & $5.157 \mathrm{e}-15$ & $5.328 \mathrm{e}-15$ & $4.658 \mathrm{e}-15$ & $3.990 \mathrm{e}-15$ \\
\hline
\end{tabular}

Convergence study of the relative error in the mean magnetic field components (normalized to rms-values) and solenoidal constraint: (a) without flux correction, (b) with mapped fluxes.

In the following paragraph we analyze the epicyclic mode. As a real-life test case we have also performed simple MRI simulations with initially vertical magnetic field of zero net-flux.

\subsection{Conservation of epicyclic energy}

By performing two-dimensional shearingsheet simulations the error in the epicyclic energy is found to be independent of the mode amplitude. For constant excitation amplitude the error is growing linearly in time. From the last row of Table 3 one can see how the relative error per orbit decreases with resolution, reflecting the third-order convergence of the underlying time integration scheme. This is because due to the CFL stability condition, the timestep is linearly proportional to the spatial resolution.

As already stated, our implementation of the source terms is integral part of the third-order integration scheme. For comparison we have also tested two conventional (operator split) methods for those terms: the first method (A) is very similar to the one found in the ZEUS-code and directly (i.e. forward Euler) integrates the Coriolis forces. The second method (B) treats the Coriolis term analytically in form of a rotation of the momentum vector. By expanding the trigonometric functions one can show that method A gives a first order approximation to method B. 


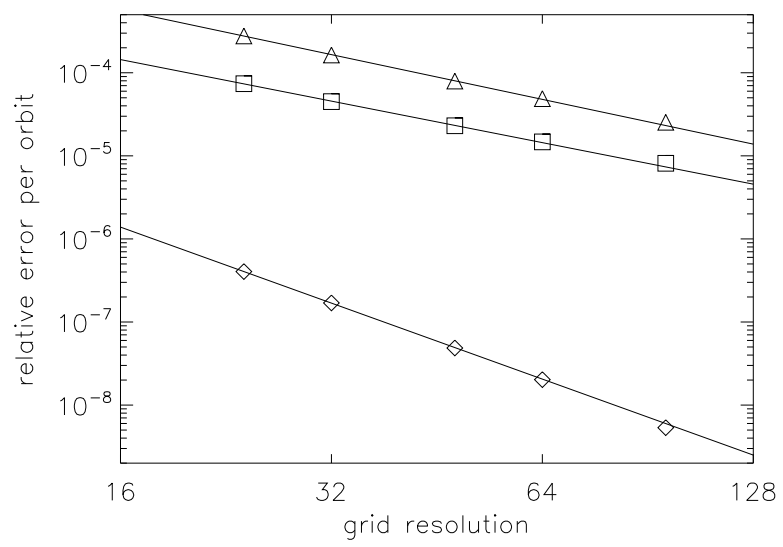

Fig. 2. Convergence of relative errors in epicyclic mode energy for method A (triangles), method B (squares), and the unsplit method (diamonds). Lines show least square fits with (logarithmic) slopes $-1.79,-1.66$, and -3.04 .

\begin{tabular}{|l|c|c|c|c|c|c|}
\hline resolution & & $24^{2}$ & $32^{2}$ & $48^{2}$ & $64^{2}$ & $96^{2}$ \\
\hline \hline method A & a & $2.776 \mathrm{e}-04$ & $1.637 \mathrm{e}-04$ & $7.943 \mathrm{e}-05$ & $4.877 \mathrm{e}-05$ & $2.535 \mathrm{e}-05$ \\
\hline & b & 0.032180 & 0.023989 & 0.015896 & 0.011886 & 0.007900 \\
\hline method B & a & $7.387 \mathrm{e}-05$ & $4.497 \mathrm{e}-05$ & $2.301 \mathrm{e}-05$ & $1.473 \mathrm{e}-05$ & $8.135 \mathrm{e}-06$ \\
\hline & b & 0.013782 & 0.010275 & 0.006810 & 0.005093 & 0.003385 \\
\hline unsplit & a & $4.045 \mathrm{e}-07$ & $1.695 \mathrm{e}-07$ & $4.862 \mathrm{e}-08$ & $2.023 \mathrm{e}-08$ & $5.345 \mathrm{e}-09$ \\
\hline & b & $1.285 \mathrm{e}-09$ & $2.468 \mathrm{e}-10$ & $8.284 \mathrm{e}-09$ & $5.652 \mathrm{e}-09$ & $4.505 \mathrm{e}-09$ \\
\hline & c & $4.038 \mathrm{e}-07$ & $1.695 \mathrm{e}-07$ & $4.874 \mathrm{e}-08$ & $1.747 \mathrm{e}-08$ & $2.587 \mathrm{e}-09$ \\
\hline
\end{tabular}

Table 3

Error growth-rates (a) and oscillation amplitudes (b) of the operator-split methods A (direct integration of Coriolis-forces) and B (exact rotation of momentum vectors). For the unsplit method we also show a simple linear fit (c).

Both methods (in contrast to the unsplit one) lead to oscillations, with frequency $2 \Omega$, in the epicyclic energy. We fit the resulting curves with a function $f(t)=a t+b \sin (2 \Omega t)$. Table 3 shows the oscillation amplitudes and linear growth-rates in the relative error as a function of resolution. For reference we also include the fits for the unsplit scheme which show negligible oscillations. Figure 2 compares the rates of convergence for the described methods, clearly favoring the unsplit approach. 

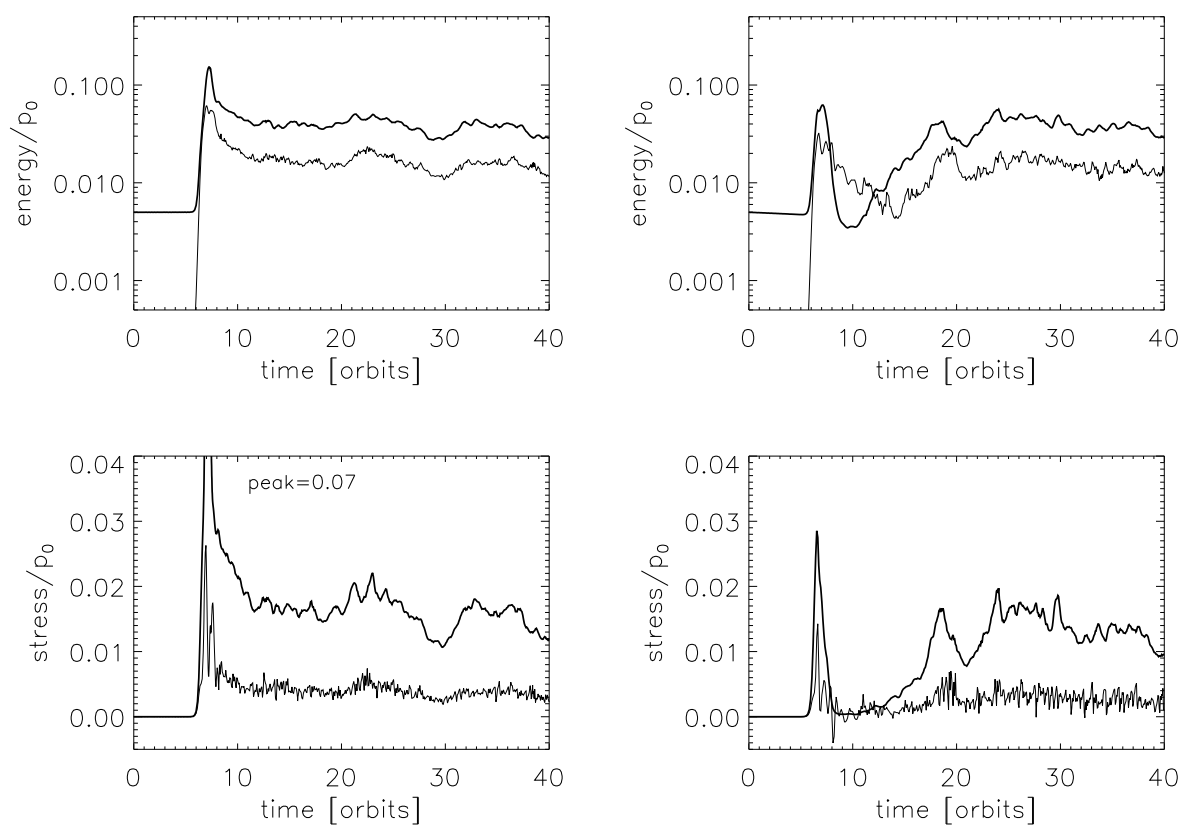

Fig. 3. Time evolution of volume averaged kinetic (thin lines) and magnetic (thick lines) energy density (top) and $R \phi$-components of Reynolds- and Maxwell-stresses (bottom) for the non-conservative scheme (left) and the conservative scheme (right).

\subsection{MRI with vertical field of zero net-flux}

The most prominent application for the shearingbox model, of course, is the magneto-rotational instability (MRI). We chose model parameters according to previous simulations [14] with the old (non-conservative) NIRVANA-code, that is very similar to the widely used ZEUS-code. For simplicity we neglect stratification in this paper. A more sophisticated model, including stratification and radiative cooling has been implemented and will be employed in future work to explore MRI under conditions suitable to the interstellar medium.

For now we use non-dimensional quantities, i.e., density is set to unity while the pressure is $p=0.5 \times 10^{-6}$, such that the sound speed over the box dimension matches the angular velocity $\Omega=10^{-3}$. The initial magnetic field is purely vertical and varies as $\mathbf{B}=B_{0} \sin \left(\pi x / L_{x}\right) \hat{\mathbf{z}}$, resulting in a vanishing net vertical flux. The field amplitude $B_{0}=1.121 \times 10^{-7}$ corresponds to a plasma parameter of $\beta=100$ at the peaks of the sine-profile.

We apply a box geometry of $[-0.5,0.5] \times[0,4] \times[-2,2]$ with a resolution of $64 \times 128 \times 128$ grid cells and perform computations on the new and the old code version to compare the conservative vs. the non-conservative scheme. The results are directly compared in Figure 3. The saturated stresses and energies are of comparable magnitude although the initial growth of the instability and 

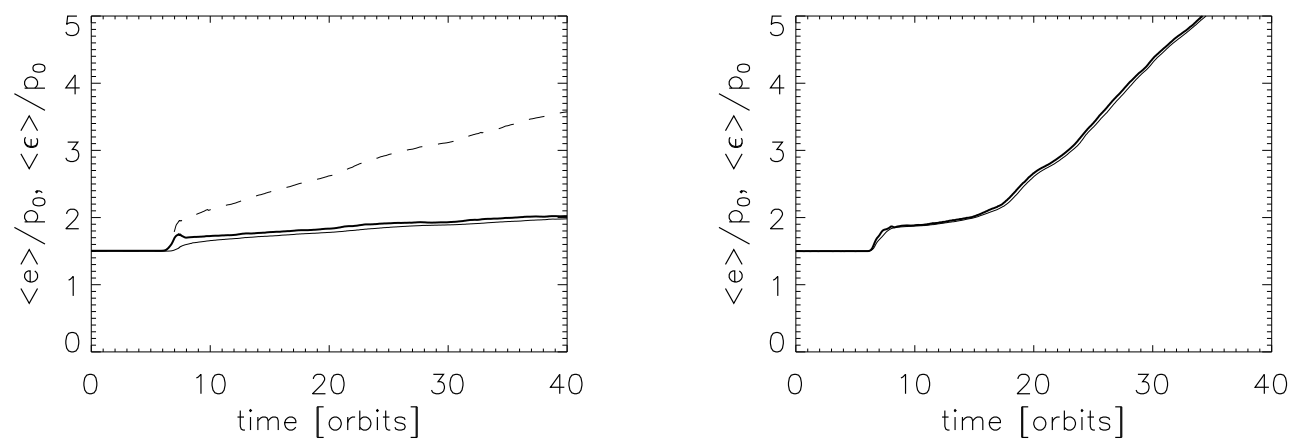

Fig. 4. Time evolution of volume averaged total (thick lines) and thermal (thin lines) energy for the non-conservative scheme (left) and the conservative scheme (right). For the former we also plot the work done by the boundary conditions (dashed line). All quantities are normalized by the initial gas pressure $p_{0}$.

the breakdown of the channel-solution into chaotic turbulence differs quite a bit. The breakdown of the linear solution is related to parasitic instabilities that seem to be resolved differently in the two codes. The weaker initial peak for the conservative scheme is consistent with the results in [6]. Saturation amplitudes are compatible with earlier results $[1,14,6]$.

Although the codes compare quite well with respect to the kinematic quantities (see Figure 3), there is a major difference concerning the thermalization of the extracted turbulent energy. As can be seen in the right panel of Figure 4 the conservative code efficiently transfers the released energy into thermal energy, which leads to a rapid heating of the gas. The non-conservative code shows much less heating. Compared to the work exerted by the boundary-stresses ${ }^{4}$, that is shown as a dashed line (on top of the total energy) in the left panel of Figure 4, there is a considerable amount of energy lost.

\section{Conclusions}

This paper described the implementation of a shearingbox environment for the flux-conservative/constraint-transport MHD code NIRVANA. To our knowledge this is the first time such an implementation has been developed for a central-type numerical scheme. We showed that shift-periodic boundary conditions accounting for a prescribed linear shear flow across the computational domain can be handled within a conservative framework. This comes about by a proper modification of the hydrodynamical numerical fluxes with the mappings of $y$-momentum flux and total energy flux solely expressed in terms of known mapped fluxes and the velocity offset of the background shear flow. Such mappings are, in principle, independent of the underlying numerical

$\overline{4}$ This quantity is obtained by accumulating the rhs of Eq. (27). 
scheme, provided it is given in flux-conservation form.

In contrast, our proposed mappings of the (face-centered) electric field fluxes contain explicit information on the magnetic field components at the boundary as well as on the characteristic speeds used by the numerical scheme. Nevertheless, we demonstrated by numerical experiment that such a mapping is divergence-free, exactely conserves the $x$ - and $z$-component of mean magnetic field and the corresponding $y$-component up to truncation error but, obviously, is scheme-dependent. An alternative method would be to directly map the edge-centered electric field fluxes, as those are the quantities which enter the constraint-transport equations. However, initial investigations of that approach have not been proven successful to date.

\section{Acknowledgements}

The MRI-computations were performed on the Sanssouci PC-cluster of the Astrophysical Institute Potsdam. This work was supported by Deutsche Forschungsgemeinschaft (DFG).

\section{References}

[1] J.F. Hawley, C.F. Gammie, S.A. Balbus, Atsrophys. J. 440 (1995) 742.

[2] S.A. Balbus, J.F. Hawley, Rev. of Mod. Phys. 70/1 (1998) 1-53.

[3] S.A. Balbus, Annual Rev. of A\&A 41 (2003) 555-597.

[4] S. E. Caunt, M. J. Korpi, A\&A. 369 (2001) 706.

[5] C.R. Evans, J.F. Hawley, Astrophys. J. 332 (1998) 659.

[6] T.A. Gardiner, J.M. Stone, AIP Conference Proceedings 784 (2005) 475.

[7] S. Gottlieb, C.-W. Shu, E. Tadmor, SIAM Rev., 43 (2001) 89-112.

[8] A. Kurganov, S. Noelle, G. Petrova, SIAM J. Sci. Comput. 23 (2001) 707.

[9] B. van Leer, J. Comput. Phys. 23 (1977) 276.

[10] G. Tóth, J. Comput. Phys. 161 (2000) 605.

[11] U. Ziegler, J. Comput. Phys. 196 (2004) 393.

[12] U. Ziegler, Comp. Phys. Commun. 170 (2005) 153.

[13] U. Ziegler, Astron. Astrophys. 435 (2005) 385.

[14] U. Ziegler, G. Rüdiger, A\&A 356 (2000) 1141. 